\title{
Carotid artery intima-media thickness is closely related to impaired left ventricular function in patients with coronary artery disease: a single-centre, blinded, non-randomized study
}

\author{
Kristin Evensen ${ }^{1,5}$, Sebastian Imre Sarvari ${ }^{2,4,5}$, Ole Morten Rønning ${ }^{3,5}$, Thor Edvardsen ${ }^{2,4,5}$ and David Russell ${ }^{1,5^{*}}$
}

\begin{abstract}
Background: Atherosclerosis is the underlying cause of the majority of myocardial infarctions and ischemic strokes. Carotid intima-media thickness (IMT) is a surrogate measure of atherosclerotic cardiovascular disease. Left ventricular (LV) function can be accurately assessed by 2D speckle-tracking strain echocardiography (2D-STE). The aim of this study was to assess the relationship between carotid IMT and LV dysfunction assessed by strain echocardiography in patients with coronary artery disease (CAD).
\end{abstract}

Methods: Thirty-one patients with symptoms of CAD were examined with coronary angiography, cardiac echocardiography and carotid ultrasound. Layer-specific longitudinal strains were assessed from endo-, mid- and epicardium by 2D-STE. LV global longitudinal strain (LVGLS) was averaged from 16 longitudinal LV segments in all 3 layers. LVGLS results were compared with coronary angiography findings in a receiver operating curve (ROC) to determine the cut-off for normal and pathological strain values. The calculated optimal strain value was compared to maximal carotid IMT measurements.

Results: The ROC analysis for strain versus coronary angiography was: area under curve (AUC) $=0.91(95 \% \mathrm{Cl} 0.80-1.0)$, cut-off value for endocardial LVGLS: $-16.7 \%$. Further analyses showed that increased carotid IMT correlated with low absolute strain values $(p=0.006)$ also when adjusted for hypertension, smoking, hyperlipidemia, diabetes and $\mathrm{BMI}(\mathrm{p}=0.02)$.

Conclusions: In this study increased carotid IMT values were associated with decreased LV function assessed by strain measurements. These findings support the use of carotid IMT measurements to predict the risk of coronary heart disease.

Keywords: Longitudinal strain, Coronary artery disease, Carotid intima-media thickness, Atherosclerosis, Ultrasound

\section{Background}

The measurement of carotid intima-media thickness (IMT) has long been regarded as a method which can be used to evaluate the presence of generalized atherosclerotic arterial disease [1,2]. In some reports carotid IMT values have been related to left ventricular (LV) hypertrophy and function $[3,4]$. One study of patients with carotid disease

\footnotetext{
* Correspondence: david.russell@medisin.uio.no

'Department of Neurology, Oslo University Hospital, Rikshopitalet, Postboks 4950 Nydalen, 0424, Oslo, Norway

${ }^{5}$ University of Oslo, Oslo, Norway

Full list of author information is available at the end of the article
}

showed a possible prediction of minimal cardiovascular resistance in patients with probable CAD [5]. LV function can be assessed with echocardiography using an ejection fraction and wall motion score index, but this method is highly operator dependent. A more reliable method that could detect early LV dysfunction would therefore be of great clinical value. Two-dimensional speckle-tracking echocardiography (2D-STE) is a semi-automated quantitative technique for the assessment of strain in the LV and is a well validated method [6,7].

Strain is an intrinsic mechanical property which measures myocardial systolic function more accurately and 
has the ability to detect early pathological changes better than conventional cavity-based echocardiographic measurements [8-10].

The LV wall is a 3-dimensional continuum of myocardial fibers with an inner right-handed helix (endocardium), a middle circular layer and an outer left-handed helix (epicardium). The endocardial layer is the most susceptible to ischemic injury. Layer-specific 2D-STE allows the assessment of LV function in all 3 layers.

Carotid IMT may be used to assess the risk for CAD $[1,2]$. Ischemia due to CAD is one of the causes of decreased LV function and there is therefore a possibility that carotid IMT may be used to assess LV function in these patients. The aim of this study was to investigate a possible correlation between IMT and LV function, assessed using strain echocardiography, in patients with CAD.

\section{Methods}

\section{Patient population}

Between September 2010 and January 2012, 31 consecutive patients with suspected CAD were assessed using B-mode ultrasound of the carotid arteries, coronary angiography, cardiac echocardiography and 2D-STE in the endo-, mid-, and epicardium. Inclusion criteria were one of the following: a history of typical or atypical angina or positive ECG stress testing. Exclusion criteria were: age $<18$ years, acute coronary syndrome (ST-elevation or non-ST elevation myocardial infarction) in the preceding 3 months, concomitant significant disease or non-cardiological therapy which could affect cardiac remodelling or function, left bundle-branch block, severe valvular dysfunction, atrial fibrillation, sustained severe arrhythmia, or any condition which interfered with the patients` ability to comply.

The study was approved by the Regional Ethic Committee (REK), Norway and subjects provided written informed consent.

\section{Carotid ultrasound}

Patients were examined in the supine position with the head angled approximately 45 degrees towards the contralateral side. IMT measurements were synchronized with the QRS complex on the ECG. Measurements were made in each carotid artery at the peak of the R-wave where the lumen is widest. IMT was defined as the distance between the lumen-intima and media-adventitia borders of the vessel identified as a double line pattern in a longitudinal image [11]. IMT was measured in the carotid arteries on both sides in 3 segments (Figures 1 and 2):

1. In the far wall of the common carotid artery (CCA) $1 \mathrm{~cm}$ proximal to the bifurcation over an area of $1 \mathrm{~cm}$ and in 3 different projections: lateral, posterior and anterior.
2. In the far wall of the carotid bifurcations (BIF) over an area of $1 \mathrm{~cm}$ in the lateral position.

3. In the far wall of the proximal internal carotid arteries (ICA) immediately distal to the bifurcation over an area of $1 \mathrm{~cm}$ in the lateral projection.

This gave a total of 10 IMT measurements in each patient. We used the highest maximum IMT in the CCA, BIF and ICA from either side of the neck for further analyses. Plaque diameter was included in the IMT measurements if present in the areas of interest. The carotid ultrasound examinations were carried out according to the American Society of Echocardiography guidelines [12], using a General Electric Vivid 7 ultrasound instrumentation with a linear M12L probe (14 MHZ) (General Electric, Horten, Norway).

\section{Coronary angiography}

All patients underwent coronary angiography. The assessment of CAD was performed by visual estimates in each single stenosis with multiple projections, avoiding overlap of side branches and foreshortening of relevant coronary stenoses. Coronary occlusion was defined as TIMI flow 0 or 1 , while significant coronary artery stenosis was considered as $\geq 50 \%$ reduction of vessel diameter in at least one major coronary artery.

\section{Two-dimensional echocardiography}

Echocardiographic studies were performed with a commercially available system (ARTIDA, Toshiba Medical Systems Corporation, Tokyo, Japan). Digital routine grey scale 2D cine loops from 3 consecutive heart beats were obtained at end-expiratory apnoea from 3 standard apical views (4chamber, 2-chamber, and long-axis). Frame rates were $47 \pm$ $5 \mathrm{~Hz}$ for the grey scale imaging. The echocardiographic data were analysed blinded for all clinical information.

\section{Two-dimensional speckle-tracking strain echocardiography} Myocardial function by strain was evaluated on a frameby-frame basis by the automatic tracking of acoustic markers (speckles) throughout the cardiac cycle. The endocardial borders were traced in the end-systolic frame of the $2 \mathrm{D}$ images from the 3 apical views for analyses of longitudinal endocardial, mid-myocardial and epicardial strain (Figure 3). Peak systolic longitudinal strain from 3 layers was assessed using off-line software (Toshiba Medical Systems Corporation, Tokyo, Japan) in 16 longitudinal segments. All segmental values were averaged to global longitudinal strain (GLS). Segments that failed to track properly were manually adjusted by the operator. Any segments that subsequently failed to track were excluded.

\section{Statistical analysis}

Data were presented as mean \pm standard deviation (SD), numbers and percentages respectively. Student's t-test 


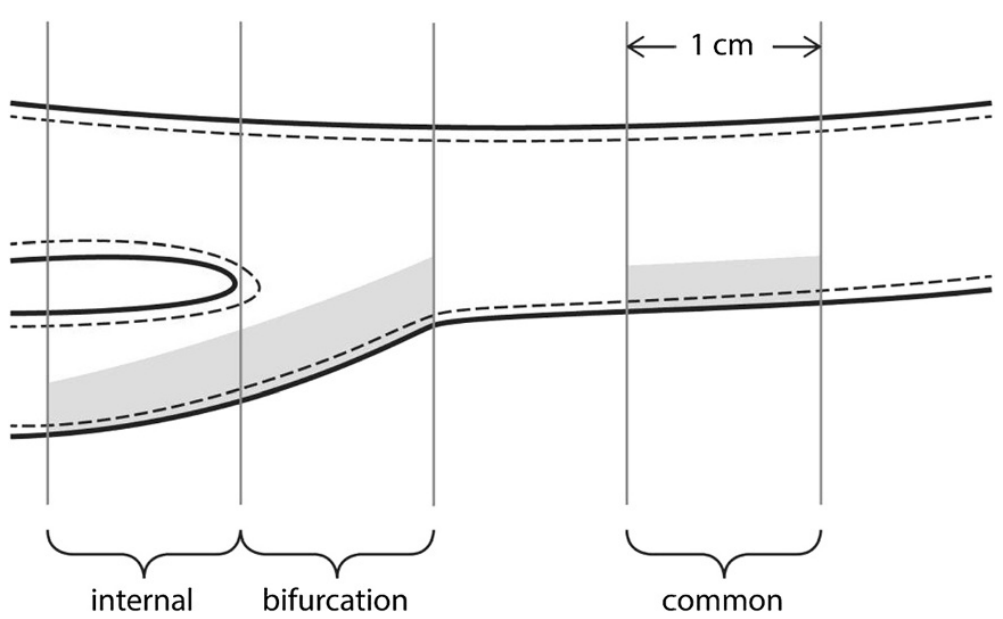

Figure 1 Drawing of a carotid artery showing the location (in grey) for the measurements of intima-media thickness (continuous-stippled line) in our study.

(continuous variables) was used to determine differences between two groups. We used multiple linear regressions to determine the relationship of carotid IMT with hypertension, smoking, hyperlipidemia, diabetes and BMI. (SPSS version 18, SPSS Inc, Chicago, Ill). The areas under the receiver operating characteristics (ROC) curves (AUC) were calculated for endocardial, mid-myocardial, epicardial and carotid IMT. The values closest to the upper left corner of the ROC curves determined optimal sensitivity and specificity for the ability of endocardial, mid-myocardial, epicardial and carotid IMT to predict the presence of significant CAD. Sensitivity and specificity was calculated using openepi.com (Open Source Epidemiologic Statistics for Public Health, Version 2.3.1.).

\section{Results}

Thirty- one patients took part in the study. Two patients were excluded due to poor echocardiographic image quality. Strain analyses were therefore performed in 29 patients.

The age of the patients ranged from 53 to 75 years. There were $81 \%$ males in the study. Baseline characteristics of the patients are shown in Table 1.

A total of $20(65 \%)$ patients had significant CAD ( $\geq 50 \%$ stenosis). Eight of these patients had an occlusion of a major coronary artery) and 11 (35\%) had a nonsignificant CAD ( $<50 \%$ stenosis). There was a significant difference in endocardial and mid-myocardial LVGLS between the patients with significant coronary stenosis and the patients with non-significant coronary stenosis. Layerspecific LVGLS compared to the coronary angiography findings are shown in Table 2.

Left ventricular Ejection Fraction (LVEF) was: mean \pm SD; $61 \% \pm 6 \%$ for the total group of patients. Patients with normal endocardial strain had EF values of: mean $\pm \mathrm{SD}$; $62 \% \pm 6.0 \%$ and patients with reduced endocardial

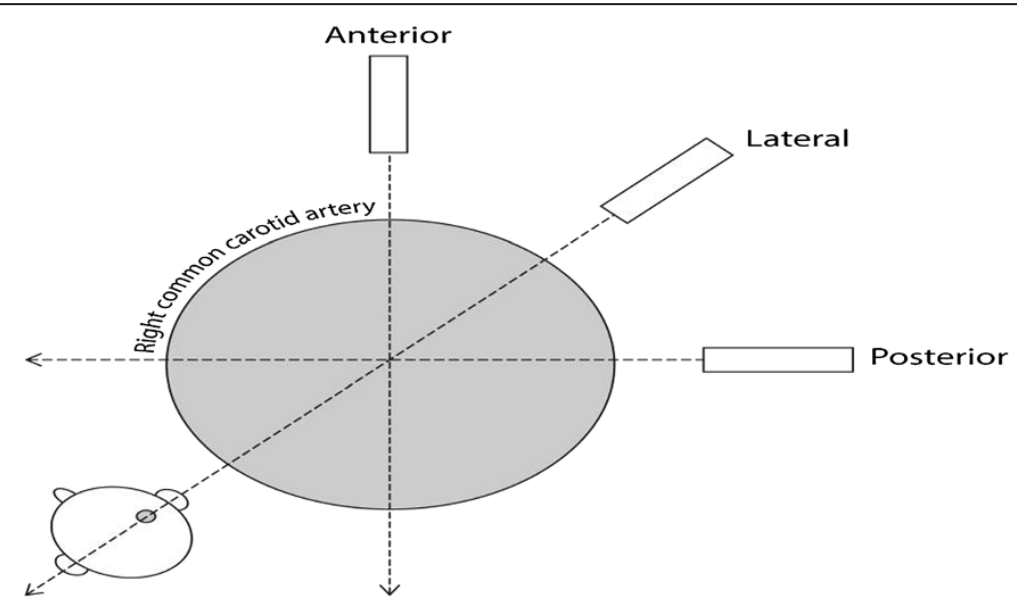

Figure 2 Drawing of the right common carotid artery showing the probe orientations for the ultrasound examinations. 


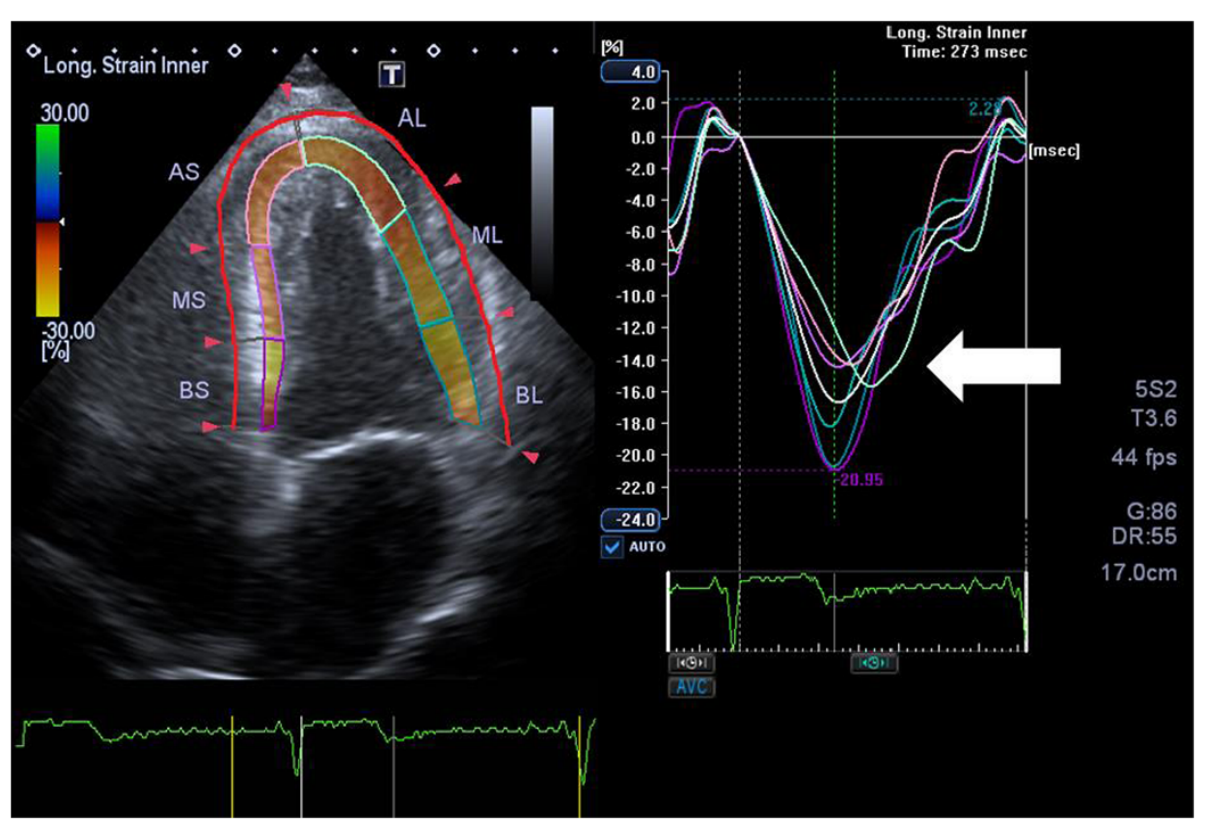

Figure 3 Endocardial longitudinal strain study of a patient with significant coronary artery disease. The automatic strain analysis in a patient with angina and significant left anterior descending (LAD) artery stenosis. The apical four-chamber view showes reduced colour coded subendocardial strain values segments supplied by the left LAD artery. Colour-coding from yellow to green indicates strain from $+30 \%$ to $-30 \%$. Yellow $=$ normal strain. Brown = areas with reduced strain. On the right strain curves for the 6 subendocardial segments are presented. The white arrow shows reduced strain values of $-14 \%$ in the curves representing the segments supplied by the $L A D$ artery. $A L=$ apicolateral; $A S=a p i c o s e p t a l ; B L=$ basolateral; $\mathrm{BS}=$ basoseptal; $\mathrm{ML}=$ midlateral; $\mathrm{MS}=$ midseptal.

strain mean \pm SD; $58 \% \pm 4.8 \%$. This difference was not significant $(\mathrm{p}=0.091)$.

Figures 4a-d shows ROC analyses of layer specific strain parameters and the ability of IMT measurements to identify patients with significant CAD. Maximum carotid IMT ranged between $1 \mathrm{~mm}$ and $2.88 \mathrm{~mm}$ for the whole group of patients including carotid plaques in the IMT measurements. For the group with normal endocardial strain values, defined by the cut-off strain value of -16.7 , carotid IMT was $1.2 \mathrm{~mm} \pm 0.2$ (mean \pm SD) and for the group with reduced endocardial strain values the carotid IMT value was $1.7 \mathrm{~mm} \pm 0.5$ (mean $\pm \mathrm{SD}$ ). Independent samples t-test comparing the two groups was statistically significant $(p=0.006)$. There was significant correlation between endocardial LVGLS and maximum carotid IMT $(p=0.006)$. The results of linear regression analyses which incorporated adjustment for known risk factors of atherosclerosis (hypertension, smoking, hyperlipidemia, diabetes and $\mathrm{BMI})$ were made and showed significant results $(\mathrm{p}=0.02)$.

\section{Discussion}

In this study we found a significant correlation between increased carotid IMT and LV dysfunction. We also found associations between significant CAD assessed by coronary angiography and reduced endocardial and mid- myocardial function assessed by 2D speckle-tracking strain echocardiography.

The cut-off value for endocardial strain was approximately $-16.7 \%$ whereas normal strain values are approximately $-20 \%$ [13]. We had no gold standard with regard to normal and abnormal 2D-STE values which could be used for the ROC curves comparing carotid IMT versus 2D-STE. We therefore calculated a cut-off value for pathological endocardial strain measurements. We found affection of LV function in the endocardial and midmyocardial layers but not in the epicardial layer. This finding is in accordance with the fact that, reduced myocardial function

\section{Table 1 Patient characteristics}

\begin{tabular}{lc}
\hline Number of patients, $\mathbf{n}$ & $\mathbf{3 1}$ \\
\hline Age (range) & $64(53-75)$ \\
Males, n (\%) & $25(81)$ \\
TIA and ischemic stroke, n (\%) & 0 \\
Heart attack (former), n (\%) & $5(16)$ \\
Diabetes, n (\%) & $5(16)$ \\
Hypertension, n (\%) & $17(55)$ \\
Hyperlipidemia, n (\%) & $12(39)$ \\
Smoking, $n(\%)$ & $5(16)$ \\
BMl & $28 \pm 4$ \\
\hline
\end{tabular}

$\mathrm{TIA}=$ transient ischemic attack; $\mathrm{BMI}$ = body mass index. 
Table 2 Layer-specific global longitudinal strain of the left ventricle compared to coronary angiography findings

\begin{tabular}{|c|c|c|c|}
\hline & $<50 \%$ stenosis $(n=11)$ & $\geq 50 \%$ stenosis $(n=18)$ & p-value \\
\hline Endocardial LVGLS & $-19.5 \pm 2.2$ & $-15.0 \pm 2.7$ & 0.001 \\
\hline Midmyocardial LVGLS & $-15.4 \pm 1.6$ & $-13.1 \pm 2.6$ & 0.011 \\
\hline Epicardial LVGLS & $-12.8 \pm 1.9$ & $-11.6 \pm 2.2$ & 0.169 \\
\hline
\end{tabular}

Left ventricular global strain (LVGLS) is in mean \pm SD.

usually starts in the endocardial layer and this layer is the most susceptible layer for ischemic injury [14].

There are few studies investigating the association between carotid disease and early left ventricular injury but there are some findings which correspond to the present study. The correlation between LV dysfunction and increased carotid IMT was found in one study of individuals free of clinical CAD, where greater carotid IMT was associated with changes of myocardial strain, measured by tagged MRI [15]. Mizuguchi et al. found that LV relaxation measured by 2D-STE was associated with carotid arterial atherosclerosis in particular related to sclerosis, measured by carotid arterial stiffness. They did not find this correlation for carotid IMT and LV strain [16].

The assessment of LV function is challenging due to its very complex deformation pattern. All imaging modalities
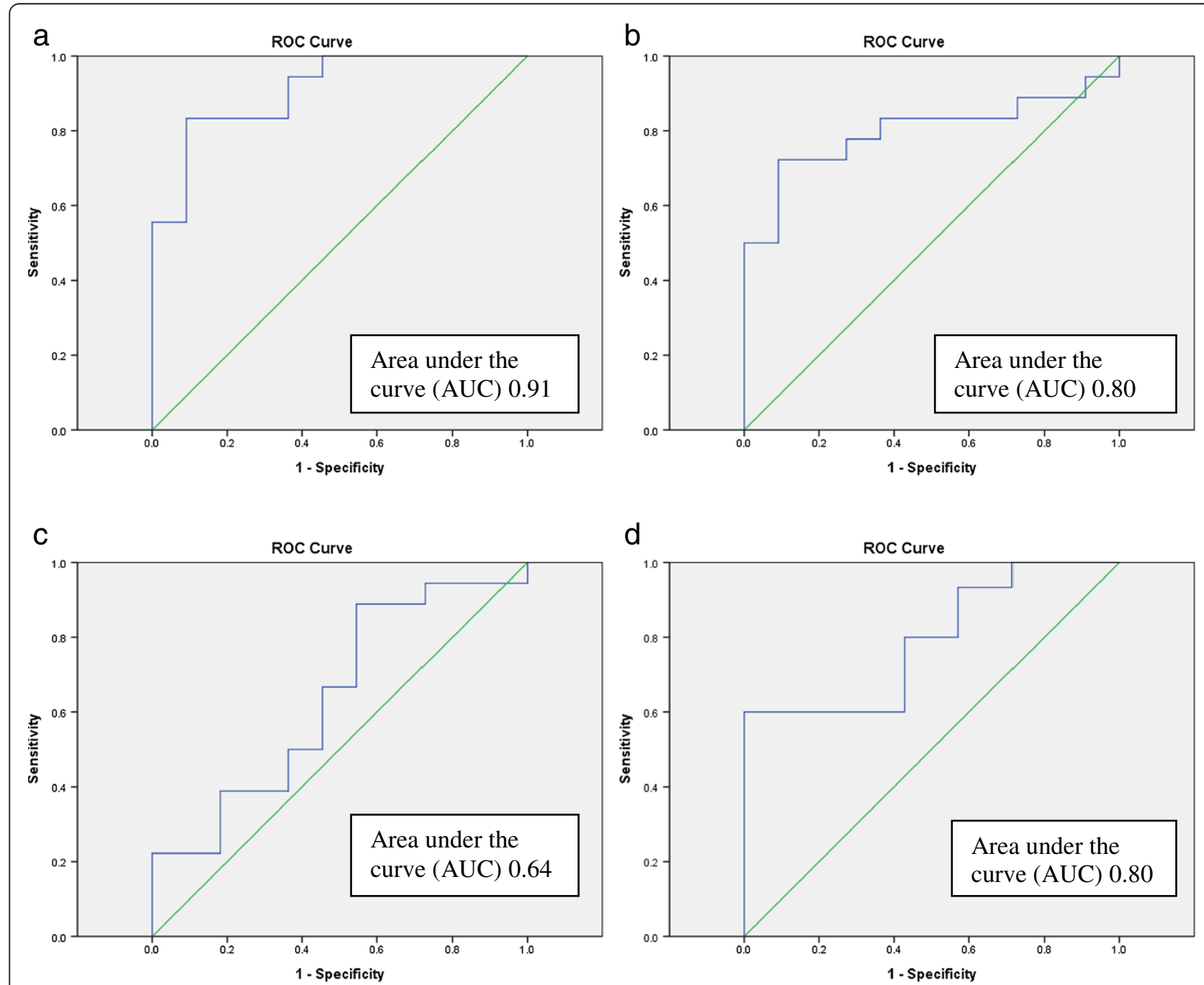

Figure 4 ROC curves for coronary angiography and carotid IMT versus ventricular strain. a: Coronary angiography versus endocardial 2D-STE. b: Coronary angiography versus mid-myocardial 2D-STE. c: Coronary angiography versus epicardial 2D-STE. d: Average maximum Carotid IMT versus endocardial 2D-STE. 
have shortcomings in this regard. However, although myocardial strain measures are only a component of LV function, it has been demonstrated to be an accurate and reproducible measure in several studies [6,7].

All patients in our study had symptoms of CAD or risk factors for atherosclerosis. It is known that structural and functional changes in the carotid arteries occur with normal aging. However, the presence of cardiovascular risk factors accelerate these changes [17-19].

It is well known that carotid intima-media thickness and plaques have different pathophysiological mechanisms. However, both carotid IMT and plaques are well-known indicators of increased risk for cerebro- and cardiovascular disease $[20,21]$. We therefore included the diameter of the small number of plaques when detected in the area of interest.

In a study of patients with hypercholesterolemia and preserved LVEF, 1 year treatment with a statin improved regional LV systolic and diastolic function measured by strain [22]. Carotid stiffness was lower after statin therapy, but there was no difference in carotid IMT before and after treatment with a statin. This is probably due to the fact that the annual rate of IMT progression is lower than the resolution of carotid ultrasound $(\approx 0.3 \mathrm{~mm})$. The presence of increased IMT may lead to detection of very early cardiac dysfunction which may benefit from cardiovascular risk management.

The results of this study support the few previous studies which have examined a possible association between carotid IMT and LV strain. B-mode ultrasound of the carotid artery is a non-invasive, inexpensive and widely used examination and may therefore be an additional appropriate tool for the assessment of possible CAD. An ultrasound examination of the carotid arteries is mandatory in all cases with cerebrovascular disease. A more detailed examination which includes IMT measurements in patients with cardiovascular risk factors may therefore be of value when estimating the risk of subclinical coronary artery disease.

\section{Conclusion}

In this study increased carotid IMT values were associated with decreased left ventricular function. These findings support the use of carotid IMT measurements to predict the risk of coronary heart disease.

\section{Abbreviations}

AUC: Area under the curve; BIF: Bifurcation; BMI: Body mass index CAD: Coronary artery disease; CCA: Common carotid artery; Cl: Confidence interval; ECG: Electrocardiography; GLS: Global longitudinal strain; ICA: Internal carotid artery; IMT: Intima-media thickness; LV: Left ventricle; LVGLS: Left ventricular global longitudinal strain; MRI: Magnetic resonance imaging; NPV: Negative predictive value; PPV: Positive predictive value; ROC: Receiver operating characteristics; SD: Standard deviation; TIA: Transitoric ischemic attack; 2D-STE: Two-dimensional speckle- tracking strain echocardiography.

\section{Competing interests}

The authors declare that they have no competing interests.

\section{Authors' contributions}

KE carried out the carotid ultrasound of the patients in the study, drafted the manuscript and performed the statistical analyses. SIS carried out the strain measurements in the patients, coordination of the study participants and participated in drafting of the manuscript. OMR participated in drafting of the manuscript. TE conceived the study and revised the manuscript. DR conceived the study and revised the manuscript. All authors read and approved of the final manuscript.

\section{Acknowledgement}

Are Hugo Pripp for statistical support.

\section{Author details}

${ }^{1}$ Department of Neurology, Oslo University Hospital, Rikshopitalet, Postboks 4950 Nydalen, 0424, Oslo, Norway. ²Department of Cardiology, Oslo University Hospital, Rikshospitalet, Oslo, Norway. ${ }^{3}$ Department of Neurology, Medical Division, Akershus University Hospital, Lorenskog, Norway. ${ }^{4}$ Institute for Surgical Research, Oslo University Hospital, Rikshospitalet, Oslo, Norway. ${ }^{5}$ University of Oslo, Oslo, Norway.

Received: 23 May 2014 Accepted: 12 September 2014

Published: 29 September 2014

\section{References}

1. Geroulakos G, O'Gorman DJ, Kalodiki E, Sheridan DJ, Nicolaides AN: The carotid intima-media thickness as a marker of the presence of severe symptomatic coronary artery disease. Eur Heart J 1994, 15(6):781-5.

2. O'Leary DH, Polak JF, Kronmal RA, Savage PJ, Borhani NO, Kittner SJ, Tracy R, Gardin JM, Price TR, Furberg CD: Thickening of the carotid wall.

A marker for atherosclerosis in the elderly? Cardiovascular Health Study Collaborative Research Group. Stroke 1996, 27(2):224-31.

3. Cuspidi C, Lonati L, Sampieri L, Pelizzoli S, Pontiggia G, Leonetti G, Zanchetti A Left ventricular concentric remodelling and carotid structural changes in essential hypertension. J Hypertens 1996, 14(12):1441-6.

4. Linhart A, Gariepy J, Giral P, Levenson J, Simon A: Carotid artery and left ventricular structural relationship in asymptomatic men at risk for cardiovascular disease. Atherosclerosis 1996, 127(1):103-12.

5. Danad I, Raijmakers PG, Kamali P, Harms HJ, De Haan S, Lubberink M, Van Kuijk C, Hoekstra OS, Lammertsma AA, Smulders YM, Heymans MW, Tulevski II, Van Rossum AC, Knaapen P: Carotid artery intima-media thickness, but not coronary artery calcium, predicts coronary vascular resistance in patients evaluated for coronary artery disease. Eur Heart J Cardiovasc Imaging 2012, 13(4):317-23.

6. Sarvari SI, Haugaa KH, Zahid W, Bendz B, Aakhus S, Aaberge L, Edvardsen T: Layer-specific quantification of myocardial deformation by strain echocardiography may reveal significant CAD in patients with non-ST-segment elevation acute coronary syndrome. JACC CardiovasC Imaging 2013, 6(5):535-44.

7. Yeon SB, Reichek N, Tallant BA, Lima JA, Calhoun LP, Clark NR, Hoffman EA, Ho KK, Axel L: Validation of in vivo myocardial strain measurement by magnetic resonance tagging with sonomicrometry. J Am Coll Cardiol 2001, 38(2):555-61.

8. Haugaa KH, Smedsrud MK, Steen T, Kongsgaard E, Loennechen JP, Skjaerpe T, Voigt JU, Willems R, Smith G, Smiseth OA, Amlie JP, Edvardsen T: Mechanical dispersion assessed by myocardial strain in patients after myocardial infarction for risk prediction of ventricular arrhythmia. JACC CardiovasC Imaging 2010, 3(3):247-56.

9. Sarvari SI, Haugaa KH, Anfinsen OG, Leren TP, Smiseth OA, Kongsgaard E, Amlie JP, Edvardsen T: Right ventricular mechanical dispersion is related to malignant arrhythmias: a study of patients with arrhythmogenic right ventricular cardiomyopathy and subclinical right ventricular dysfunction. Eur Heart J 2011, 32(9):1089-96.

10. Sjoli B, Orn S, Grenne B, Ihlen H, Edvardsen T, Brunvand H: Diagnostic capability and reproducibility of strain by Doppler and by speckle tracking in patients with acute myocardial infarction. JACC CardiovasC Imaging 2009, 2(1):24-33.

11. Touboul PJ, Hennerici MG, Meairs S, Adams H, Amarenco P, Bornstein N, Csiba L, Desvarieux M, Ebrahim S, Hernandez Hernandez R, Jaff M, Kownator S, 
Naqvi T, Prati P, Rundek T, Sitzer M, Schminke U, Tardif JC, Taylor A, Vicaut E, Woo KS: Mannheim carotid intima-media thickness and plaque consensus (2004-2006-2011). An update on behalf of the advisory board of the 3rd, 4th and 5th watching the risk symposia, at the 13th, 15th and 20th European Stroke Conferences, Mannheim, Germany, 2004, Brussels, Belgium, 2006, and Hamburg, Germany, 2011. Cerebrovasc Dis 2012. 34(4):290-6.

12. Stein JH, Korcarz CE, Hurst RT, Lonn E, Kendall CB, Mohler ER, Najjar SS, Rembold CM, Post WS: Use of carotid ultrasound to identify subclinical vascular disease and evaluate cardiovascular disease risk: a consensus statement from the American Society of Echocardiography Carotid Intima-Media Thickness Task Force. Endorsed by the Society for Vascular Medicine. J Am Soc Echocardiogr 2008, 21(2):93-111.

13. Leitman M, Lysiansky M, Lysyansky P, Friedman Z, Tyomkin V, Fuchs T, Adam D, Krakover R, Vered Z: Circumferential and longitudinal strain in 3 myocardial layers in normal subjects and in patients with regional left ventricular dysfunction. J Am Soc Echocardiogr 2010, 23(1):64-70.

14. Reimer KA, Lowe JE, Rasmussen MM, Jennings RB: The wavefront phenomenon of ischemic cell death. 1. Myocardial infarct size vs duration of coronary occlusion in dogs. Circulation 1977, 56(5):786-94.

15. Fernandes VR, Polak JF, Edvardsen T, Carvalho B, Gomes A, Bluemke DA, Nasir K, O'Leary DH, Lima JA: Subclinical atherosclerosis and incipient regional myocardial dysfunction in asymptomatic individuals: the Multi-Ethnic Study of Atherosclerosis (MESA). J Am Coll Cardiol 2006, 47(12):2420-8.

16. Mizuguchi $Y$, Tanaka H, Oishi Y, Miyoshi H, Emi S, Ishimoto T, Nagase N, Oki T: Predictive value of associations between carotid arterial sclerosis and left ventricular diastolic dysfunction in patients with cardiovascular risk factors. J Am Soc Echocardiogr 2007, 20(7):806-12.

17. Kawasaki T, Sasayama S, Yagi S, Asakawa T, Hirai T: Non-invasive assessment of the age related changes in stiffness of major branches of the human arteries. Cardiovasc Res 1987, 21(9):678-87.

18. Gil-Nunez AC, Villanueva JA: Advantages of lipid-lowering therapy in cerebral ischemia: role of HMG-CoA reductase inhibitors. Cerebrovasc Dis 2001, 11(Suppl 1):85-95.

19. Mitchell GF: Pulse pressure, arterial compliance and cardiovascular morbidity and mortality. Curr Opin Nephrol Hypertens 1999, 8(3):335-42.

20. Lorenz MW, Markus HS, Bots ML, Rosvall M, Sitzer M: Prediction of clinical cardiovascular events with carotid intima-media thickness: a systematic review and meta-analysis. Circulation 2007, 115(4):459-67.

21. O'Leary DH, Polak JF, Kronmal RA, Manolio TA, Burke GL, Wolfson SK Jr: Carotid-artery intima and media thickness as a risk factor for myocardial infarction and stroke in older adults. Cardiovascular Health Study Collaborative Research Group. N Engl J Med 1999, 340(1):14-22.

22. Mizuguchi Y, Oishi Y, Miyoshi H, luchi A, Nagase N, Oki T: Impact of statin therapy on left ventricular function and carotid arterial stiffness in patients with hypercholesterolemia. Circ J 2008, 72(4):538-44.

doi:10.1186/1476-7120-12-39

Cite this article as: Evensen et al: Carotid artery intima-media thickness is closely related to impaired left ventricular function in patients with coronary artery disease: a single-centre, blinded, non-randomized study. Cardiovascular Ultrasound 2014 12:39.

\section{Submit your next manuscript to BioMed Central and take full advantage of:}

- Convenient online submission

- Thorough peer review

- No space constraints or color figure charges

- Immediate publication on acceptance

- Inclusion in PubMed, CAS, Scopus and Google Scholar

- Research which is freely available for redistribution 